\title{
Nutritional deficit in miliary tuberculosis: prognostic value
}

\section{To the Editors:}

KIM et al. [1] have previously shown the prognostic importance of nutritional deficit in the development of acute respiratory failure and further outcome in miliary tuberculosis (MTB). There are a few points that need to be discussed further so that its relevance is properly understood.

Under 4-point Nutritional Risk Score, KIM et al. [1] have used severe lymphocytopenia and hypocholesterolaemia as the parameters of poor nutritional status. MTB is characterised by compartmentalisation of lymphocytes at the site of inflammation (lymphocytic alveolitis), leading to their reduced number in peripheral blood [2]. Moreover, total lymphocyte count has not been found to be a suitable marker of malnutrition in the elderly [3]. In addition, keeping the varied presentations and leukocyte counts in MTB in mind, use of severe lymphocytopenia as a parameter does not seem justified.

The role of hypocholesterolaemia as a nutrition status parameter is an area of active research and has not been well proven. Low serum cholesterol levels are also caused by inflammatory mediators during active infection [4] and hence, may not truly depict nutritional deficit. Literature on the association between low serum cholesterol levels and tuberculosis outcome is also lacking.

Malnutrition is the most common cause of immunodeficiency. Nutrition status is a nonspecific parameter that critically determines the outcome of all infections and is not specific to MTB. Animal experiments have shown that malnutrition leads to decreased immunological response to infection, and particularly diminished lymphocyte stimulation and cytokine secretion, leading to poor outcome [5]. Other risk factors, such as presence of meningismus [6], hyponatraemia [7], elevated transaminase levels [8] and adrenal suppression, may specifically predict poor outcome in MTB.

Nutritional deficit has a complex interaction with infection. It not only increases susceptibility to infection but also determines its outcome. Infection may also precipitate nutritional deficiency as in tuberculosis. Initial presentation and severity of miliary tuberculosis may be a better predictor of disease outcome. However, nutritional deficiency can be a confounding factor, and hence should always be looked for and managed along with anti-tubercular therapy in all forms of tuberculosis.

\section{Aggarwal and P.R. Mohapatra}

Dept of Pulmonary Medicine, Government Medical College and Hospital, Chandigarh, Punjab, India.

\section{STATEMENT OF INTEREST}

None declared.

\section{REFERENCES}

1 Kim DK, Kim HJ, Kwon SY, et al. Nutritional deficit as a negative prognostic factor in patients with miliary tuberculosis. Eur Respir J 2008; 32: 1031-1036.

2 Sharma SK, Pande JN, Singh YN, et al. Pulmonary function and immunologic abnormalities in miliary tuberculosis. Am Rev Respir Dis 1992; 145: 1167-1171.

3 Kuzuya M, Kanda S, Koike T, Suzuki Y, Iguchi A. Lack of correlation between total lymphocyte count and nutritional status in the elderly. Clin Nutr 2005; 24: 427-432.

4 Bentz MH, Magnette J. Hypocholesterolemia during the acute phase of an inflammatory reaction of infectious origin. 120 cases. Rev Med Interne 1998; 19: 168-172.

5 Cegielski JP, McMurray DN. The relationship between malnutrition and tuberculosis: evidence from studies in humans and experimental animals. Int J Tuberc Lung Dis 2004; 8: 286-298.

6 Gelb AF, Leffler C, Brewin A, Mascatello V, Lyons HA. Miliary tuberculosis. Am Rev Respir Dis 1973; 108: 1327-1333.

7 Sharma SK, Mohan A, Pande JN, Prasad KL, Gupta AK, Khilnani GC. Clinical profile, laboratory characteristics and outcome in miliary tuberculosis. QJM 1995; 88: 29-37.

8 Maartens G, Willcox PA, Benatar SR. Miliary tuberculosis: rapid diagnosis, hematologic abnormalities, and outcome in 109 treated adults. Am J Med 1990; 89: 291-296.

DOI: $10.1183 / 09031936.00084708$

\section{To the Editors:}

We read with great interest the recently published informative article by KIM et al. [1] on the prognostic value of nutritional deficit in miliary tuberculosis (TB). Using a nutritional risk score comprising of four factors (low body mass index, hypoalbuminaemia, hypocholesterolaemia and severe lymphocytopenia), the authors demonstrated the independent and major prognostic values of nutritional status on both acute respiratory failure and 90-day mortality among 56 patients with miliary TB [1]. However, with the observational nature and retrospective design of the study, it might be difficult to pinpoint the exact cause and effect relationship between nutrition status and TB severity/outcome. 